\title{
The Interaction of Water with Glycine: A Combined Inelastic Neutron Scattering and Raman Spectra Studies
}

\author{
P. ZHANG ${ }^{a, b}, \mathrm{Y} . \mathrm{ZHANG}^{b}, \mathrm{~S} \cdot \mathrm{HAN}^{a}, \mathrm{Q} \cdot \mathrm{YAN}^{b} \mathrm{AND}^{\mathrm{J}} \cdot \mathrm{LI}^{c}$ \\ ${ }^{a}$ Department of Space Science \& Applied Physics \\ Shandong University at Weihai, Weihai 264209, China \\ ${ }^{b}$ State Key Laboratory for Magnetism, Institute of Physics \\ Chinese Academy of Sciences, Beijing 100080, China
}

${ }^{c}$ Department of Physics, The University of Manchester, Manchester M60 1QD, UK

The vibrational dynamics of water around glycine was investigated by using Raman spectroscopy and inelastic neutron scattering. Experiments of deuterated glycine versus deuterium were performed as comparison. The study shows that for glycine, the exchange of proton-deuteron on the active $\mathrm{NH}_{3}^{+}$side was easy, whereas there was hardly exchange on the $\mathrm{CH}_{2}$ side. Comparing different proportion of glycine vs. water molecules we obtained that the presence of water hardly changes the main features of glycine illustrating its hydrophobic character. The intralayer hydrogen bonds of glycine crystal are difficult to be replaced due to its stronger bond than water.

PACS numbers: 36.20.Ng, 87.64.Je, 25.40.Fq, 82.30.Rs

\section{Introduction}

The simplest aminoacid, glycine, has frequently been used as a prototype for larger systems. In the crystalline form, glycine molecules exist in the dipolar, i.e., zwitterionic form $\left(\mathrm{NH}_{3}^{+} \mathrm{CH}_{2} \mathrm{COO}^{-}\right)$[1]. It has monoclinic structure (space group symmetry $P 2_{1} / n$ ) with four formula units per unit cell [2-4]. In this structure, the dipolar glycine molecules are linked by two relatively short $\mathrm{N}-\mathrm{H} \cdots \mathrm{O}$ hydrogen bonds (with the relative bonds length of $\mathrm{H} \cdots \mathrm{O}$ equal to $1.728 \AA$ and $1.832 \AA$ ) to form layers parallel to the $a c$ plane, the layers are connected, two by two, by weaker bifurcated $\mathrm{N}-\mathrm{H} \cdots \mathrm{O}$ bonds (the two bond distances are $2.121 \AA$ and $2.365 \AA$ ), forming antiparallel double layers. There are no N-H. . O bonds between the different double layers.

The interaction between glycine (neutral or ionic form) and water has been widely studied by theoretical methods using different approaches. Ramaekers et 
al. have reported hydrated glycine experiment on gas phase in isolated state by IR spectroscopy [5]. However, experiment on glycine from crystalline state to hydrated form has never been published as far as we know. In this contribution we report results on the interaction of water clusters with glycine by the Raman spectroscopy and inelastic neutron scattering (INS). Experiments on deuterated glycine were also performed for comparison.

\section{Experimental techniques}

The aminoacid samples were purchased from Sigma. Samples referred to in this paper as "dry" were obtained by drying glycine under a vacuum at $80^{\circ} \mathrm{C}$. The water content was determined by weight difference and expressed throughout this paper as mol proportion versus dry sample. The samples were kept under the vacuum to avoid the adsorption of atmospheric water and saturated after $12 \mathrm{~h}$, before experiment. The deuterated glycine was made by diluting sample in deuterium solution and then recrystallized by slow evaporation using a vacuum drier.

The Raman scattering measurements were performed with a multichannel modular triple Raman system (JY-T6400) with confocal microscopy. A long focus 50X microscope objective lens was used to focus the laser beam on the sample surface and to collect the scattered light. The excitation wavelength at $488 \mathrm{~nm}$ of an $\mathrm{Ar}^{+}$ion laser was used in this experiment. The laser power on the sample was kept with $3 \mathrm{~mW}$ and typical spectrum acquisition time was $200 \mathrm{~s}$. All measurements were taken at room temperature.

The neutron scattering experiments were performed on the TOSCA instrument at the ISIS pulsed spallation neutron source of the Rutherford-Appleton Laboratory at the temperatures below $20 \mathrm{~K}$. TOSCA is an indirect geometry time-of-flight spectrometer with an optimal energy range of $0-4000 \mathrm{~cm}^{-1}(0-500 \mathrm{meV})$. The best results are normally obtained below $2000 \mathrm{~cm}^{-1}(250 \mathrm{meV})$. The resolution of the spectrometer is determined by a number of factors, but for practical purposes it can be considered at $2 \%$ of the energy transfers $[6,7]$.

\section{Results and discussion}

The assignments of most of the modes are found to be consistent with the spectra reported earlier [8-10]. Positions of Raman bands characterizing glycine molecule and even (to a certain extent) their relative intensities are not very sensitive to the environmental changes (i.e. water content in our case). As shown in Fig. 1, there are two $\mathrm{NH}_{3}^{+}$rocking modes, of which one is weak at $1109 \mathrm{~cm}^{-1}$, and the other is medium at $1140 \mathrm{~cm}^{-1}$. Only relative intensities of the two peaks changed slightly with the increase in water content. As for $\mathrm{NH}_{3}^{+}$asymmetric deformation $\left(1506 \mathrm{~cm}^{-1}, 1570 \mathrm{~cm}^{-1}\right)$ and symmetric deformation modes $\left(1670 \mathrm{~cm}^{-1}\right)$, almost no changes can be observed with the increase in water concentrations. 
Raman spectra of glycine with different concentrations of water in the range from $1600 \mathrm{~cm}^{-1}$ to $4000 \mathrm{~cm}^{-1}$ are presented in Fig. 2. Since the intralayer $\mathrm{N}-\mathrm{H} \cdots \mathrm{O}$ hydrogen bond lengths are similar to water, our experiments show that the intralayer $\mathrm{N}-\mathrm{H} \cdots \mathrm{O}$ hydrogen bonds are difficult to be broken by the polarized water molecules. A weak broad band observed at $3145 \mathrm{~cm}^{-1}$ has been identified as N-H stretching modes [11, 12]. From the known correlation between the $\mathrm{N}-\mathrm{H}$ stretch frequencies and various $\mathrm{N}-\mathrm{H} \cdots \mathrm{O}$ distances, the value of $3145 \mathrm{~cm}^{-1}$ corresponds to the weak interlayer hydrogen bond [13]. Figure 2 shows that the intensity at $3145 \mathrm{~cm}^{-1}$ decreases when $3 \mathrm{~mol}$ water was added, meanwhile the COO- group stretching modes from $2500 \mathrm{~cm}^{-1}$ to $3000 \mathrm{~cm}^{-1}$ increased [14]. It is suggested that when $3 \mathrm{~mol}$ water molecules soaked into glycine crystal, the

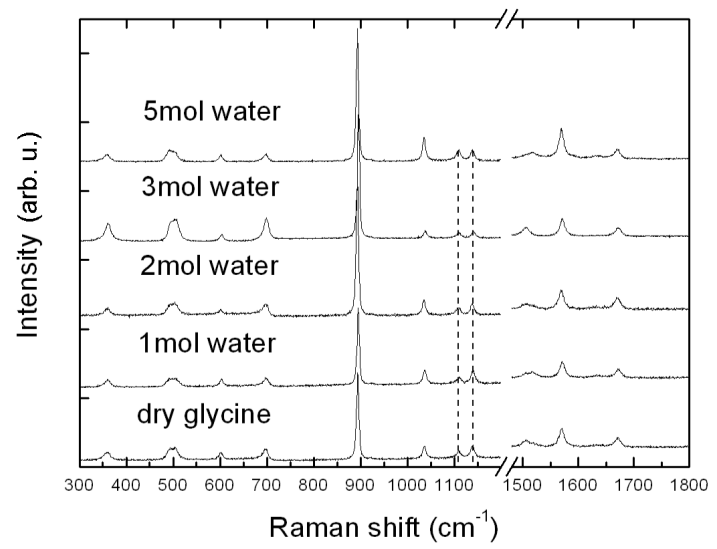

Fig. 1. Raman spectra of glycine with different concentration of $\mathrm{H}_{2} \mathrm{O}$ region from $300 \mathrm{~cm}^{-1}$ to $1800 \mathrm{~cm}^{-1}$.

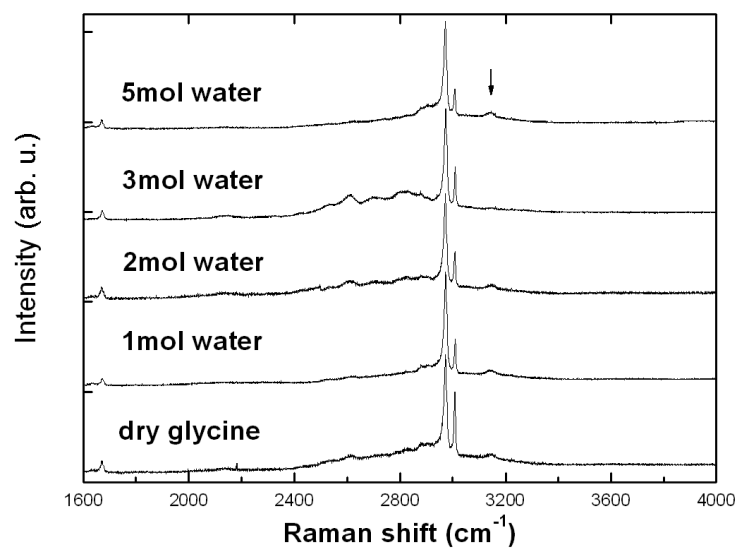

Fig. 2. Raman spectra of glycine with different concentration of $\mathrm{H}_{2} \mathrm{O}$ region from $1600 \mathrm{~cm}^{-1}$ to $4000 \mathrm{~cm}^{-1}$. 
layers of glycine crystal may be separated by polarized water molecule and new hydrogen bonds between glycine within layer and water molecules were formed.

The Raman spectra for the deuterated glycine are presented in Fig. 3. As a comparison, it is clearly shown that the intensity of $\mathrm{N}-\mathrm{H}$ vibrational modes described above decrease while the $\mathrm{ND}_{3}$ rocking at $1001 \mathrm{~cm}^{-1}$ increases. However, the unchanged $\mathrm{C}-\mathrm{H}$ vibrational modes indicate that the exchange of protondeuteron on the active $\mathrm{NH}_{3}^{+}$side was relatively easier than the $\mathrm{H}$ on the $\mathrm{CH}_{2}$ sides. As for the deuterated samples, the $\mathrm{N}-\mathrm{D}$ rocking modes at $1001 \mathrm{~cm}^{-1}$ shift towards high energy transfer at $1015 \mathrm{~cm}^{-1}$ indicating that the ambient of $\mathrm{ND}_{3}^{+}$ group changed, which resembles the interaction of glycine with water [8].

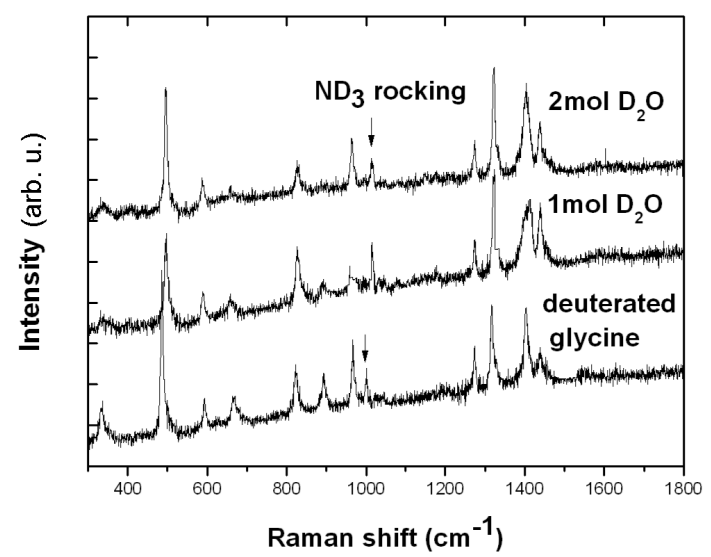

Fig. 3. Raman spectra of deuterated glycine with different concentration of $\mathrm{D}_{2} \mathrm{O}$ region from $300 \mathrm{~cm}^{-1}$ to $1800 \mathrm{~cm}^{-1}$.

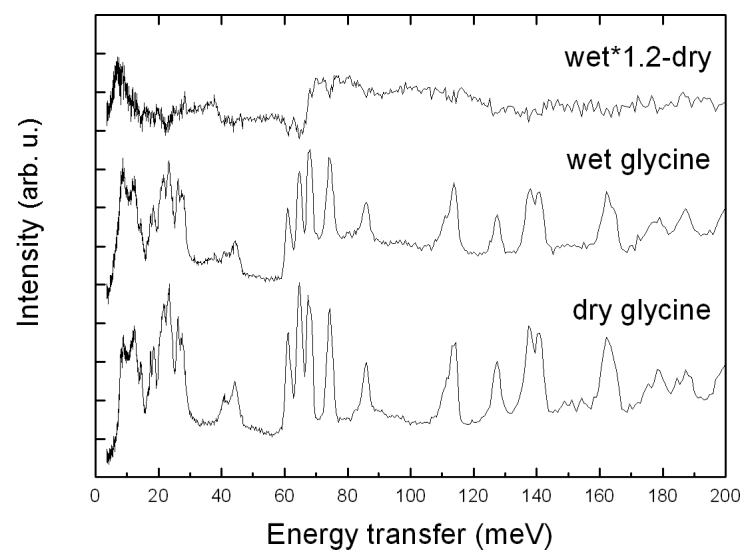

Fig. 4. Inelastic neutron scattering spectra of glycine on TOSCA.

The TOSCA spectra are presented in Fig. 4, which are in good agreement with the Raman spectra. The characteristic peaks of glycine with 1 mol water 


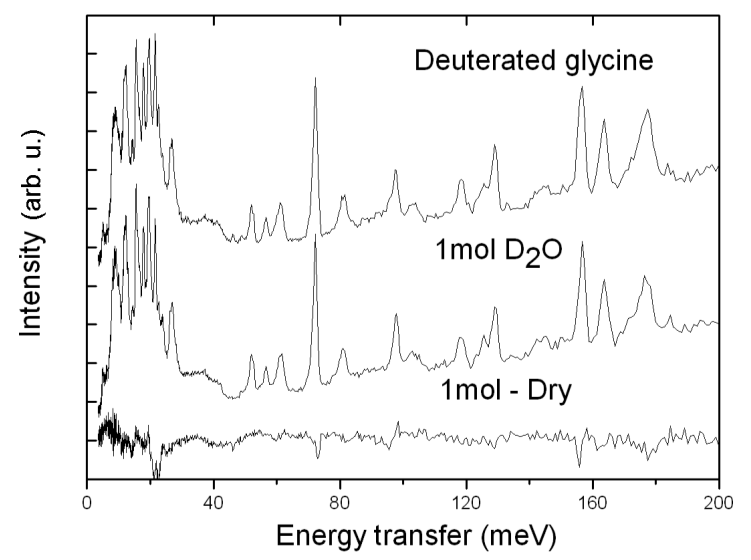

Fig. 5. Inelastic neutron scattering spectra of deuterated glycine on TOSCA.

hardly shift versus the dry sample. One can see clearly that the difference spectrum (i.e. the top curve in Fig. 4) is very similar to the INS spectrum of bulk water measured before [6]. The two sharp peaks related to the translational motion of the hydrogen bonds in water at $28.3 \mathrm{meV}$ and $37.8 \mathrm{meV}$ are outstanding $[6,15]$. The energy at $7.2 \mathrm{meV}$ is the acoustic phonon region and the energy bands from $67 \mathrm{meV}$ to $121 \mathrm{meV}$ are librations of water molecules in the bulk states. This implies that water and the aminoacids are completely separated in two separated "phases".

The deuterated spectra are presented in Fig. 5. The difference spectrum obtained by subtracting the dry spectrum from $1 \mathrm{~mol}$ wet spectrum is around the zero line due to low scattering cross-section of deuterium, hence no information was provided from the $\mathrm{D}_{2} \mathrm{O}$ water.

\section{Conclusion}

In this paper, we have reported our recent investigation of interaction between water and glycine. Corresponding spectra for deuterated glycine were also presented. Comparison of the spectra with different concentrations of water allow us to conclude that:

(1) Lower concentrations of water molecules cannot penetrate into intralayer of glycine crystal. The water around glycine appears identical to the bulk water.

(2) The hydrogen bonds may be hard to change around glycine molecule due to its stronger intermolecule hydrogen bonds, demonstrating its hydrophobic property.

(3) The exchange of proton-hydrogen on the active $\mathrm{NH}_{3}$ side was observed, whereas exchange on the $\mathrm{CH}_{2}$ sides was not observed. 


\section{Acknowledgments}

We would like to acknowledge the Institute of Physics of Chinese Academy of Sciences to offer "Outstanding Youth Fund B" to support Raman experiments. We also thank ISIS of Rutherford-Appleton Laboratory to perform TOSCA experiments.

\section{References}

[1] G.L. Perlovich, L.K. Hansen, A. Baur-Brandl, J. Therm. Anal. Calorimetry 66, 699 (2001).

[2] G. Albrecht, R.B. Corey, J. Am. Chem. Soc. 61, 1087 (1939).

[3] P.-G. Jonsson, A. Kvick, Acta Crystallogr. B 28, 1827 (1972).

[4] R.E. Marsh, Acta Crystallogr. 11, 654 (1958).

[5] R. Ramaekers, J. Pajak, B. Lambie, G. Maes, J. Chem. Phys. 120, 4182 (2004).

[6] J.C. Li, J. Chem. Phys. 105, 6733 (1996).

[7] J.C. Li, M.J. Leslie, J. Phys. Chem. 101, 6304 (1997).

[8] K. Furić, V. Mohaček, M. Bonifačić, I. Štefanić, J. Mol. Struct. 267, 39 (1992).

[9] S. Suzuki, T. Shimanouchi, M. Tsuboi, Spectrochim. Acta A 19, 1195 (1963).

[10] S.F.A. Kettle, E. Lugwisha, J. Echet, N.K. McGuire, Spectrochim. Acta A 45, 533 (1989).

[11] K. Machida, A. Kagayama, Y. Saito, Y. Kuroda, T. Uno, Spectrochim. Acta A 33, 569 (1977).

[12] H.J. Himmler, H.H. Eysel, Spectrochim. Acta 45, 1077 (1989).

[13] R.S. Krishnan, K. Krishnan, Proc. Ind. Acad. Sci. A 60, 11 (1964).

[14] D.H. Williams, I. Fleming, Spectroscopic Methods in Organic Chemistry, McGrawHill, UK 1995.

[15] J.C. Li, D.K. Ross, Nature 365, 327 (1993). 